\title{
Autologous hematopoietic stem cell transplantation for acute myeloid leukemia - single center experience
}

\author{
I. SIMANCIKOVA*, E. BOJTAROVA, M. HRUBISKO, F. FARKAS, D. HORVATHOVA, L. SOPKO, A. BATOROVA, M. MISTRIK \\ Department of Hematology and Transfusiology, Medical Faculty, Commenius University, Slovak Medical University and University Hospital \\ Bratislava, Slovakia
}

*Correspondence: simancikova@gmail.com

Received December 22, 2016 / Accepted February 8, 2017

\begin{abstract}
We aimed to determine the effect of autologous hematopoietic stem cell transplantation (auto-HSCT) on acute myeloid leukemia (AML) patients as a valid alternative therapeutic option for patients without HLA-compatible donor. This retrospective single center study included 79 patients with AML older than 18 years. In this report, we describe the patient characteristics, engraftment, toxicity of treatment, complications, overall survival, and relapse incidence of 79 patients treated chemotherapy and followed by auto-HSCT. The descriptive statistics was used, and the method of Kaplan and Meier was applied to calculate the actuarial rate of overall survival. The patients achieved an absolute neutrophile count (ANC) of $\geq 0.5 \times 109 / 1$ in between 10 to 40 days; median was 14 days after auto-HSCT. The patients achieved platelet count $\geq 20 \times 109 / 1$ in between 10 to 209 days; median was 19 days after auto-HSCT. Hundred-day mortality after autologous transplant was 6.57\% (5/76). The relapse rate was 39.5\% (32 patients) and 7 patients (8.6\%) were lost from follow-up. On the date of evaluation (April 30, 2016), 48 patients $(60.8 \%)$ were alive, including 7 (8.6\%) patients who are lost from follow-up (not responding to check-up request). The 5-year overall survival (OS) was $60.8 \%$; median overall survival was not reached. The present clinical study has demonstrated safety and efficacy of myeloablative chemotherapy followed by auto-HSCT in the treatment of AML in first remission.
\end{abstract}

Key words: acute myeloid leukemia, autologous hematopoietic stem cell transplantation, overall survival, post remission therapy

Hematopoietic stem cell transplantation (HSCT) still remains as the most efficient therapy for patients with acute leukemia. For older patients and those lacking a related HLA-compatible donor, autologous hematopoietic stem cell transplantation is a valid alternative therapeutic option [1]. Post-remission therapy in patients with acute myeloid leukemia (AML) may consist of continued chemotherapy only or transplantation using either autologous or allogeneic stem cells. Although allogeneic HSCT (allo-HSCT) is considered the preferred type of post-remission therapy in poor risk AML, the place of allo-HSCT in intermediate-risk AML is being debated and autologous HSCT is considered a valuable alternative that may be preferred in patients without minimal residual disease after induction chemotherapy [2]. We review post remission transplantation strategies using autologous stem cell transplantation. The use of auto-HSCT as consolidation therapy for adult patients with acute leukemia has declined over time. However, multiple randomized studies in the past have reported lower relapse rates after au- tologous transplantation compared with chemotherapy and lower non-relapse mortality rates compared with allogeneic transplantation. In addition, quality of life of long-term survivors is better after autologous transplantation than after allogeneic transplantation [3]. Further, recent developments may improve outcomes of autograft recipients. These include the use of intravenous busulfan and busulfan-melphalan combination, better detection of minimal residual disease (MRD) with molecular biology techniques, the introduction of targeted therapies, and post-transplant maintenance therapy. Therefore, auto-HSCT may nowadays be reconsidered for consolidation in the following patients if and when they reach a MRD-negative status: standard and at least intermediate risk acute myeloid leukemia in first CR, acute promyeloid leukemia in second $\mathrm{CR}, \mathrm{Ph}$-positive acute lymphoblastic leukemia. Conversely, patients with MRD-positive status or high-risk leukemia should not be considered for consolidation with auto-HSCT [4]. Intensive chemotherapy for acute myeloid leukemia (AML) achieves a complete hema- 
tologic remission in the majority of patients. However, despite high complete remission rates and continued antileukemic treatment as well, the majority of patients still relapse with their underlying disease. The duration of response is usually short and most of the patients will experience a leukemia relapse. Allogeneic stem cell transplantation is a therapeutic option that is curative in a significant proportion of patients, but is associated with morbidity and mortality resulting from graftversus-host disease and opportunistic infections. Moreover, not every patient has a fully matched histocompatible sibling donor or matched unrelated donor. Autologous stem cell transplantation (auto-HSCT) is an alternative approach, which was initially designed to consolidate remission in AML patients lacking a sibling donor or unfit for allo-HSCT [5]. Auto-HSCT holds several advantages including low non-relapse mortality rates, absence of graft-vs-host disease (GVHD) risk, lower incidence of late effects and better quality of life for survivors compared to allo-HSCT; concerns include high relapse rate, due to the absence of graft-vs-leukemia (GVL) effect and the theoretic possibility of graft contamination by leukemic cells [2]. Autologous stem cell transplantation has been used for the therapy of AML with disease-free survival rates ranging from $18 \%$ to $67 \%$ depending on the phase of disease, the preparative regimen, follow-up time, whether purging was used (and the method of purging) and time from achieving complete remission to transplantation. In an attempt to improve antitumor efficacy of the preparative regimen, we used the combination of busulfan and melphalan. In this report, we describe the patient characteristics, engraftment, toxicity, complications and overall survival, and relapse incidence of 79 patients treated with this regimen, followed by autologous bone marrow (BM) or (and) peripheral blood (PB) stem cell transplantation.

\section{Patients and methods}

The study was set as a retrospective single center study. All patients affected by AML older than 18 at the time of diagnosis, who received auto-HSCT between December 1994 and May 2016 were included. The total number 79 patients received first auto-HSCT during this 22-year period, median of annual number of auto-SCT performed for AML at our department was 3 , ranged from 0 to 8 (Figure1). Thirty eight patients (48\%) were transplanted in the first 10 years and 41 patients (52\%) in the last 13 years.

Patient characteristics are described in Table 1 . The median age was 44 years, with a range from 20 to 67 years. There were 44 males and 35 females. French-American-British (FAB) classification showed M0: 3, M1: 7, M2: 35, M4: 20 and M5: 7, 6 patients were classified as secondary AML after myelodysplasion syndrome and 1 patient with as bifenotypic leukemia. The white blood cell (WBC) count at diagnosis ranged from 0.78 to $393 \times 10^{9} / 1$ with average of $42.18 \times 10^{9} / 1$ and median is $14.75 \times 10^{9} / 1$. Cytogenetic analysis was without abnormalities in 34 patients, 38 patients had a variety of different

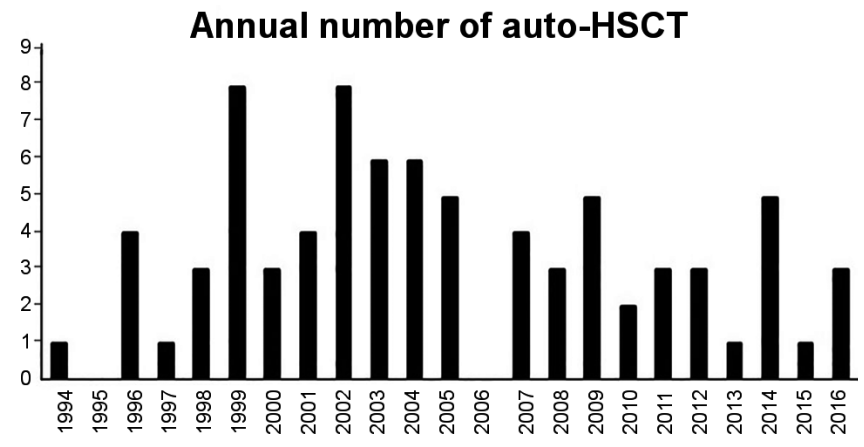

Figure 1. Annual numbers of autologous hematopoietic stem cell transplantation.

translocations and analysis was not performed in 7 patients. The patients were divided in three groups according to their risk status (Table 2).

Induction and consolidation treatment before autologous transplantation. A majority of the patients received similar remission inductions based on combination of cytarabine and anthracycline. A standard protocol, which we have been using for the last 8 years, is cytarabine 100$200 \mathrm{mg} / \mathrm{m}^{2}$ continuous intravenous infusion for 7 days plus daunorubicin $45-90 \mathrm{mg} / \mathrm{m}^{2} /$ day for 3 days (dosage depends on age and comorbidities). Before this protocol we used other protocols, for example protocol EORTC AML 12 (induction treatment containing daunorubicin, etoposide and either standard-dose cytarabine $100 \mathrm{mg} / \mathrm{m}^{2}$ per day by continuous infusion for 10 days or high-dose cytarabine $3 \mathrm{~g} / \mathrm{m}^{2}$ every 12 hours by 3 -hour infusion on days $1,3,5$, and 7) or other protocols including combination of cytarabine, anthracycline and inhibitors of topoisomerase. Six patients received two inductions. 35 patients received one consolidation therapy (protocol DIA - or Mayer - Highdose cytarabine $3 \mathrm{~g} / \mathrm{m}^{2}$ over $3 \mathrm{~h}$ every $12 \mathrm{~h}$ on days 1,3 , and 5), followed with auto-HSCT. 32 patients received two consolidation therapies (multiple protocols; mostly based on combination of high dose cytarabine, anthracycline and inhibitors of topoisomerase), followed with auto-HSCT. Other patients either received more consolidations (3-5), or after the first or second consolidation developed relapse and received reinduction protocols. In the first complete remis-

Table 1. Patient characteristics at the time of diagnosis

\begin{tabular}{lcc}
\hline No. of patients & 79 & \\
\hline Age (years) & Range 20-67 & Median 44 \\
\hline Sex (male/ female) & Male 44 & Female 35 \\
\hline \multirow{3}{*}{ FAB classification } & M1 - & M2 - 35 \\
\cline { 2 - 3 } & M4 - 20 & M5 - 7 \\
\cline { 2 - 3 } First WBC $\left(\mathbf{x} 1 \mathbf{0}^{9} / \mathbf{l}\right)$ & Range 0.78 to 393 & biphenotypic 1 \\
\hline
\end{tabular}


Table 2. Prognostic subgroups of AML based on cytogenetic and genetic lesions [6]

\begin{tabular}{|c|c|c|c|}
\hline Risk status & Cytogenetic & Molecular abnormalities & No. of patients \\
\hline Standard & $\begin{array}{l}-\mathrm{t}(8 ; 21)(\mathrm{q} 22 ; \mathrm{q} 22) \\
-\operatorname{inv}(16)(\mathrm{o} 13 . \mathrm{q} 22) \\
-\mathrm{t}(16 ; 16)(\mathrm{p} 13 . \mathrm{q} 22) \\
-\mathrm{t}(15 ; 17)\end{array}$ & $\begin{array}{l}\text { Normal cytogenetic with NPM1 mutation or } \\
\text { CEBPA mutation in absence of FLT3-TD }\end{array}$ & 9 \\
\hline Intermediate & $\begin{array}{l}- \text { Normal } \\
-\quad+8 \\
-\mathrm{t}(3 ; 5) \\
-\mathrm{t}(9 ; 11)(\mathrm{p} 22 \mathrm{q} 23) \\
- \text { Other non-defined }\end{array}$ & $\begin{array}{l}\text { c-KIT mutation with: } \mathrm{t}(8 ; 21)(\mathrm{q} 22 . \mathrm{q} 22) \text {, or } \\
\operatorname{inv}(16)(\mathrm{p} 13 . \mathrm{q} 22), \mathrm{t}(16 ; 16)(\mathrm{p} 13 . \mathrm{q} 22)\end{array}$ & 50 \\
\hline Poor & $\begin{array}{l}\text { - Complex karyotype (>3 abnormalities) } \\
\text { - MK+ } \\
-\quad-5,5 \mathrm{q}^{-} \\
-\quad-7,7 \mathrm{q}- \\
- \text { other } 11 \mathrm{qp} 23 \text { abnl [non } \mathrm{t}(9 ; 11)] \\
\text { - inv (3)(q21q26.2) } \\
-\mathrm{t}(3 ; 3)(\mathrm{q} 21 \mathrm{q} 26.2) \\
-\mathrm{t}(6 ; 9), \mathrm{t}(9 ; 22) \\
-\operatorname{abnl}(17 \mathrm{p})\end{array}$ & $\begin{array}{l}\text { High EV11 expression (with or without } 3 \mathrm{q} 26 \\
\text { cytogenetic lesion) } \\
\text { Normal cytogenetics with FLT3-ITD in absence } \\
\text { of NPM1 mutation }\end{array}$ & 13 \\
\hline
\end{tabular}

sion (CR1) we transplanted 66 patients $(83.5 \%), 12$ patients $(15.25 \%)$ were in the second complete remission (CR2), one patient $(1.25 \%)$ was in the first relapse, and one patient $(1.25 \%)$ was in progression of disease. The time from first date of diagnosis to auto-HSCT varied from 74 days to 1791 days with median 175 days.

Peripheral blood stem cell mobilization and collection. Mobilization was carried out after or during regeneration from last chemotherapy. Human recombinant G-CSF was used as the priming agent subcutaneously. The administration of G-CSF began at least 5 days before apheresis. G-CSF administration was continued daily until peripheral blood stem cell collection was completed and did not have to be withdrawn from any patients because of adverse effects. Apheresis was started and performed on consecutive days using a continuous flow blood cell separator COBE Spectra (COBE Laboratories, Lakewood, CO, USA), Spectra OPTIA apheresis system 61000 (Lakewood, CO, USA) or CS-3000 Plus (Fenwall, Round Lake, IL, USA). In all cases a preapheresis hemoglobin level $>100 \mathrm{~g} / \mathrm{l}$, a polymorphonuclear cell count $>1.5 \times 10^{9} / 1$ and a platelet count $100 \times 10^{9} / 1$ were registered. Apheresis was performed until the total number of mononuclear cells (MNC) collected reached $4 \times 10^{8}$ per kilogram of body weight until January 1995, from which

Table 3. Conditioning regimen

\begin{tabular}{lc}
\hline Conditioning regimen (n) & Number \\
\hline Busulfan + cyclophosphamide & 4 \\
Busulfan + melphalan & 70 \\
Busulfan + cyclophosphamide + etoposide & 2 \\
Busulfan + etoposide & 1 \\
Busulfan & 2 \\
\hline
\end{tabular}

time the procedure was performed until an amount of $3 \times 10^{6}$ CD $34^{+}$cells/kg were collected. The collected cell suspension was immediately frozen in a controlled-rate freezer after each apheresis using DMSO 10\% v/v as cryoprotectant and stored at $-196{ }^{\circ} \mathrm{C}$ in liquid nitrogen until use.

Bone marrow collection and processing. Minimum of $2 \times 10^{8}$ nucleated marrow cells per kilogram of body weight were collected from the posterior iliac crest. The bags of bone marrow were frozen using DMSO as cryoprotectant in a controlled-rate freezer (Cryomed, New Baltimore, MI) at $-1^{\circ} \mathrm{C}$ per minute to a temperature of $-50{ }^{\circ} \mathrm{C}$ and at $-10{ }^{\circ} \mathrm{C}$ per minute to a temperature of $-70^{\circ} \mathrm{C}$ and then transferred to the liquid phase of a liquid-nitrogen freezer.

Transplantation. Risks and benefits were explained to each patient in detail during at least two outpatient visits and again on the day of admission. Thereafter, written consent was obtained from the patients and in one case from the parent of patient, who lacks legal capacity to act of her own behalf because of mental disability (age for legal action in Slovakia is 18 years). Five patients received unmanipulated bone marrow. Sixty seven patients received peripheral blood stem cells (PNSC) and 7 patients received combination of PBSC plus bone marrow due to a low total stem cells number harvested from peripheral blood. A median of $2.72 \times 10^{6}$ (range 0.52 to 32.5) $\mathrm{CD} 34^{+}$cells were infused to the patients.

Preparative regimen. Seventy $(88.6 \%)$ patients received preparative regimen consisting of busulfan $1 \mathrm{mg} / \mathrm{kg}$ body weight orally, four times daily for 4 days, total dose $16 \mathrm{mg} / \mathrm{kg}$ administered on day -6 through day -3 , and melphalan 100$150 \mathrm{mg} / \mathrm{m}^{2}$ intravenous over 4 hours on day -2 . Busulfan with cyclophosphamide (BuCy2) conventional myeloablative conditioning was given to $4(5 \%)$ patients, BuCy2 plus etoposide to $2(2.5 \%)$ patients, busulfan monotherapy to $2(2.5 \%)$ patients, and one $(1.26 \%)$ patient received busulfan plus etoposide 
(Table 3.). The graft was infused on day 0 in all patients. At the time of autologous marrow or PBSCs administration, each bag with graft was thawed rapidly in a $37^{\circ} \mathrm{C}$ water bath and infused through a central venous catheter over 5 to 10 minutes.

Supportive care. The patients were housed in the single or double bed rooms. Reverse isolation techniques with masks and gowns were used when neutrophil counts decreased to less than $0.5 \times 10^{9} / 1$. When fever exceeded $38^{\circ} \mathrm{C}$, broad-spectrum antibiotics were instituted. Intravenous antifungals (voriconazole or echinocandin or amphotericin B) were given when fever persisted for more than 3-5 days despite appropriate antibacterial treatment. Transfusions were given to maintain hemoglobin above $90 \mathrm{~g} / \mathrm{l}$ and platelet count above $10 \times 10^{9} / 1$. All blood products were irradiated with $25 \mathrm{~Gy}$ before transfusion to avoid the risk of transfusion-related acute graft-versus-host disease induction.

Statistical analysis. The descriptive statistics was used, and the method of Kaplan and Meier was applied to calculate the actuarial rate of overall survival.

\section{Results}

Engraftment. Patients achieved an absolute neutrophil count (ANC) of $\geq 0.5 \times 10^{9} / 1$ in between 10 and 40 days, median was 14 days, average was 15 days, only one patient died before achieving an absolute neutrophil count of $0.5 \times 10^{9} / 1$. Patients achieved ANC $1 \times 10^{9} / 1$ in 9 to 39 days, median was 13 days, average was 15 days, only one patient died early, before blood count recovery. Patients achieved not transfused platelet count $\geq 20 \times 10^{9} / 1$ in between 10 to 209 days; median was 19 days, 10 patients didn't achieve platelets count above $20 \times 10^{9} / 1$. Median of patients' discharge

\section{Survival Function - overall survival}

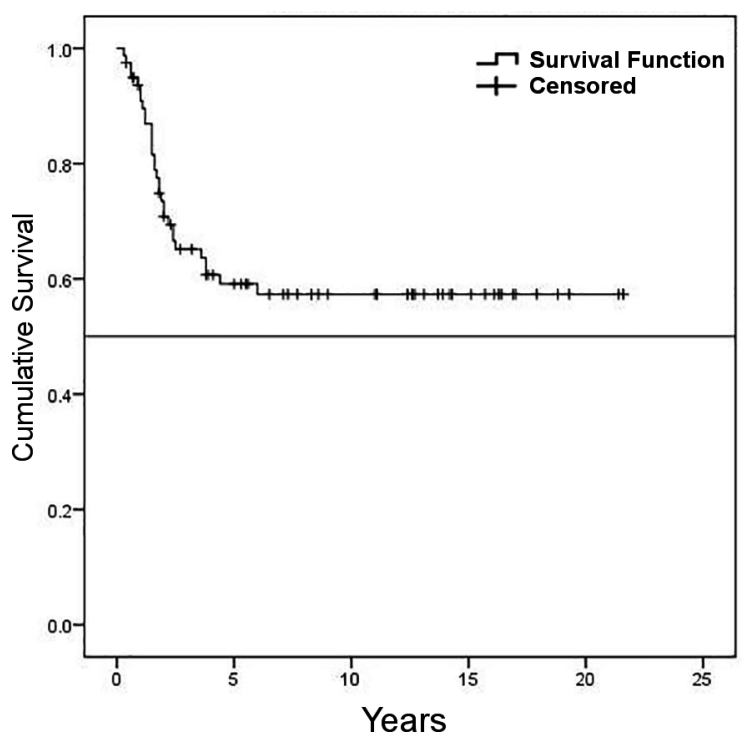

Figure 2. Overall survival. from hospital was 19 days (range from 13 to 44 days) since graft administration.

Toxicity. The combination of busulfan and melphalan was associated with the expected bone marrow aplasia in all patients. Moderate to severe mucositis was observed in a majority of patients requiring opioids analgesics for pain control. Most of the patients had at least one episode of fever. Five patients died before day 100 from auto-HSCT, 3 due to infectious complications, one patient due to hemorrhage, and one because of disease progression. Hundred-day mortality after autologous transplant was 6.57\% (5/76). Three patients were not evaluable because they had not yet achieved day 100 .

Relapse and survival. The relapse rate was 39.5\% (32/79). On the date of evaluation (April 30, 2016), 48 patients (60.8\%) were alive, including $7(8.6 \%)$ patients who are lost from follow-up (not responding to check-up request), but are alive according to health insurance database and actual report. The 5 -year overall survival (OS) is $60.8 \%$; median overall survival was not reached (Figure 2). Follow-up period of the surviving patients ranged from 115 days to 7898 days, median follow up was 1424 days and after auto-HSCT ranged from 9 to 7394 days, median follow up was of 991 days.

We also analyzed overall survival probability in 72 patients (cytogenetic analysis was not performed in 7 patients) divided in three different subgroups of patients according to cytogenetic risk status. The 5-years overall survival probability for poor risk patients (OS) was $30.8 \%$; median overall survival is 2 years (Figure 3 ). The 5 -years overall survival probability in standard risk patients was $66.7 \%$; median overall survival not reached (Figure 4 ). The 5 -years overall survival probability in intermediate risk patients (OS) was $68 \%$; median overall survival not reached (Figure 5).

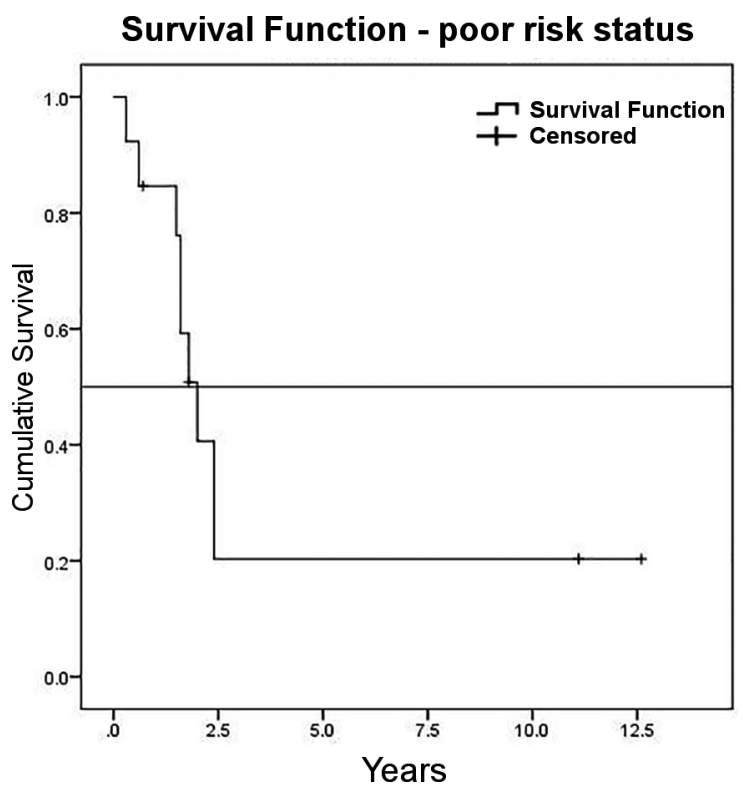

Figure 3. Overall survival of patients with poor risk cytogenetic. 


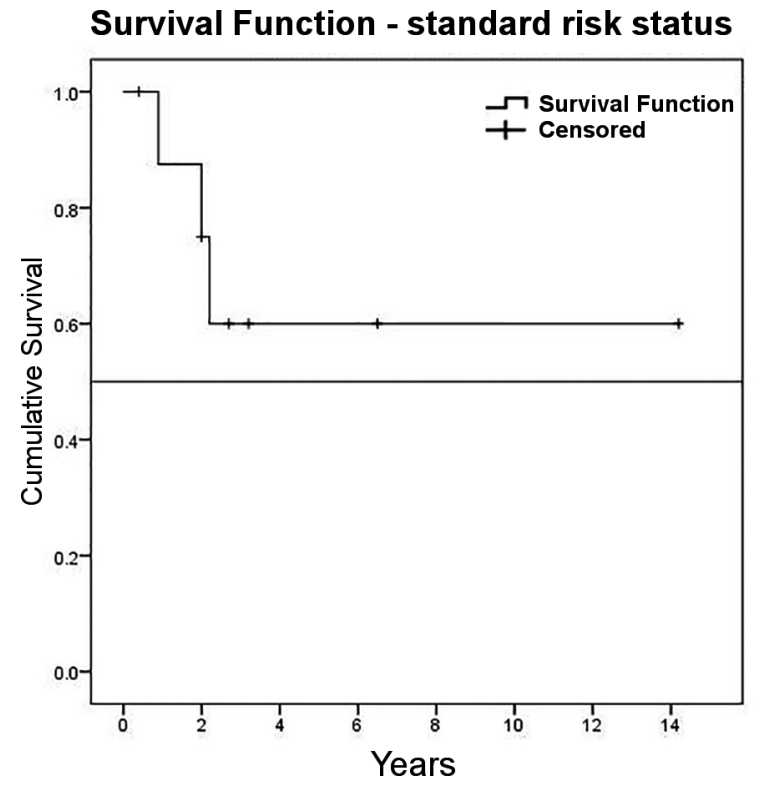

Figure 4. Overall survival of patients with standard risk cytogenetic.

Cause of failure. Thirty-one patients (39.2\%) were dead at the time of evaluation. Twenty-tree patients (29.1\%) died because of relapse disease, 1 patient died due to allogeneic transplantation complication (graft versus host disease of liver) after relapse of disease, 1 patient died due to generalized CMV infectious and encephalitis (day 656 after auto-HSCT), 3 patients $(5.1 \%)$ died due to infectious complications after auto-HSCT (last one in 2007), 1 patient died due to progression of disease right after auto-HSCT, 1 patient due to hemorrhage related to auto-HSCT and we do not have information about cause of dead of 1 patient.

\section{Discussion}

The best post-remission therapy for acute myeloid leukemia is still being discussed. The strategy depends on risk stratification, age, having HLA matched siblings or unrelated donor [7]. Several randomized trials have shown significantly better LFS for auto-HSCT compared to chemotherapy as consolidation strategy of remission in AML [8-11]. In a prospective study, Vellenga et al. [10] observed the reduced relapse rate following auto-HSCT in comparison with chemotherapy consolidation. This group reported better survival following auto-HSCT in intermediate-risk AML. Our team has studied auto-HSCT in AML using a two-step approach model based on the concept of in vivo purging [12]. We treated patients with an intensive consolidation therapy using cytarabine in combination with anthracycline and/or inhibitor of topoisomerase. Hematopoietic stem-cells were collected during hematologic recovery stimulated with G-CSF.

Consolidation chemotherapy containing intermediate-dose cytarabine in the present study may be responsible for in vivo purging. The timing of PBSC collection may be important for

\section{Survival Function - intermediate risk status}

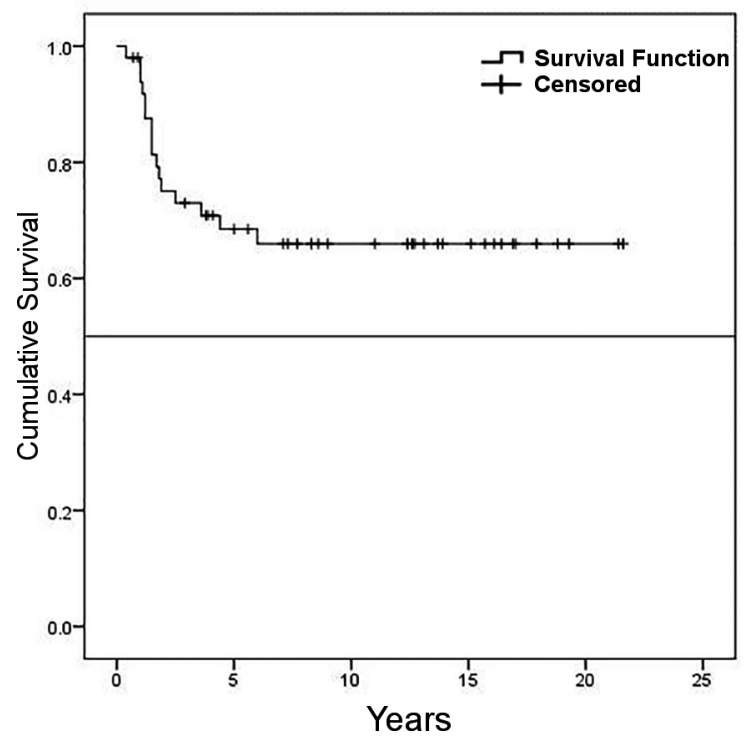

Figure 5. Overall survival of patients with intermediate risk cytogenetic.

preventing relapse. It was demonstrated that minimal residual disease in marrow and PBSC harvests could be reduced with repeated cycles of chemotherapy [13]. Based on this data, we did not collect PBSC after induction chemotherapy, but during the recovery phase after consolidation. This strategy of in vivo purging has been used to reduce the leukemic burden in the setting of auto-HSCT and has led to an improved DFS [14, 15]. Adequate consolidation of remission before auto-HSCT is the most important factor associated with continuing remission after auto-HSCT [15].

Today, peripheral blood is preferable to bone marrow as a source of stem cells. However, initial data about auto-HSCT indicated that relapse rates are as high as $60 \%$ for AML and early relapse is rather frequent when the source of cells is not bone marrow $[15,16,17,18,19]$. This may occur because the ratio of leukemic clonogenic progenitors to normal stem cells is higher in PBSC harvests than in marrow or because a large number of malignant cells are reinfused since the cell dose is higher in stem cells than in bone marrow $[15,19]$. The initial concern that the use of auto-HSCT might lead to higher relapse rates than auto-HBMT had been largely answered by larger studies, where the use of PBSC was not associated with an increased risk of relapse [15-17]. Moreover, auto-HSCT has some advantages over autologous bone marrow transplantation: auto-HSCT leads to faster hematopoietic recovery, rapid engraftment, reduced transplant related mortality, and to satisfactory outcome.

Our study confirmed rapid granulocyte recovery in patients with AML treated with myeloablative chemotherapy and auto-HCST. Engraftment after transplantation was quick with patients reaching ANC $>0.5 \times 10^{9} / 1$ at median day +14 resulting in a duration of neutropenia $\left(\mathrm{ANC}<0.5 \times 10^{9} / \mathrm{l}\right)$ of 10 days. Platelets recovered to $>20 \times 10^{9} / 1$ by day +19 . 
Despite a markedly accelerated granulocyte recovery, side effects that were noticed probably related to intensive chemotherapy and resulting in a treatment-related mortality of $6.57 \%$. However, it is similar to published data, and most studies have reported 5-10\% transplant related mortality $[16,17,14]$. Conditioning regimen was based on busulfan plus melphalan, and a median cell dose of $2.72 \times 10^{6} / \mathrm{kg}$ of body weight (range 0.52 to 32.5$) \mathrm{CD} 34+$ was administered. Gorin et al. reported a 2 -year LFS of $61 \%$ with auto-HSCT following the same busulfan plus -melphalan conditioning [20]. In our study the 5 -year overall survival probability is $60.8 \%$, and median overall survival was not reached (see Figure 2). Possible explanation include low rate of transplant-related mortality. As in other studies, cytogenetic risk status was the major determinant of outcome. OS in patients with standard and intermediate risk was significantly better than in poor risk subgroup (68\%, 66\% and $30 \%$ respectively). It confirms that cytogenetic abnormality at diagnosis has prognostic significance. The encouraging results warrant additional prospective studies to evaluate the use of auto-HSCT in the treatment of AML.

\section{Conclusion}

The present clinical study has demonstrated safety and efficacy of myeloablative chemotherapy followed by auto-HSCT in the treatment of AML in first remission. Auto-HSCT is a suitable therapeutic option for patients without HLA-compatible donor and opens transplantation treatment for older patients with comorbidities as well. Based on current understanding of AML heterogeneity it is necessary to emphasize the riskadapted approach to the AML treatment, including auto-HSCT.

\section{References}

[1] SAVANI BN, LABOPIN M, KROGER N, FINKE J, EHNINGER $G$ et al. Expanding transplant options to patients over 50 years-improved outcome after reduced intensity conditioning mismatched-unrelated donor transplantation for patients with acute myeloid leukemia: a report from the Acute Leukemia Working Party of the EBMT. Haematologica 2016; 101: 773-780. https://doi.org/10.3324/ haematol.2015.138180

[2] GORIN NC, GIEBEL S, LABOPIN M, SAVANI BN, MONTHY M et al. Autologous stem cell transplantation for adult acute leukemia in 2015: time to rethink? Present status and future prospects. Bone Marrow Transplant 2015; 50: 1495-1502. https://doi.org/10.1038/bmt.2015.179

[3] KEATING A, DASILVA G, PEREZ WS, GUPTA V, CUTLER CS et al. Autologous blood cell transplantation versus HLAidentical sibling transplantation for acute myeloid leukemia in first complete remission: a registry study from the Center for International Blood and Marrow Transplantation Research. Haematologica 2013; 98: 185-192. https://doi.org/10.3324/ haematol.2012.062059

[4] MIZUTANI M, HARA M, FUJITA H, AOKI J, KANAMORI $\mathrm{H}$ et al. Comparable outcomes between autologous and al- logeneic transplant for adult acute myeloid leukemia in first CR. Bone Marrow Transplant 2016; 51: 645-653. https://doi. org/10.1038/bmt.2015.349

[5] GORIN NC, NAJMAN A, DUHAMEL G. Autologous bonemarrow transplantation in acute myelocytic leukaemia. Lancet 1977; 309: 1050. https://doi.org/10.1016/S01406736(77)91275-2

[6] FORAN JM. New prognostic markers in acute myeloid leukemia: perspective from the clinic. Hematology Am Soc Hematol Educ Program 2010; 2010: 47-55. https://doi.org/10.1182/ asheducation-2010.1.47

[7] CORNELISSEN JJ, GRATWOHL A, SCHLENK RF, SIERRA J, BORNHAUSER $M$ et al. The European LeukemiaNet AML Working Party consensus statement on allogeneic HSCT for patients with AML in remission: an integrated-risk adapted approach. Nat Rev Clin Oncol 2012; 9: 579-590. https://doi. org/10.1038/nrclinonc.2012.150

[8] BURNETT AK, GOLDSTONE AH, STEVENS RM, HANN IM, REES JK et al. Randomised comparison of addition of autologous bone-marrow transplantation in intensive chemotherapy for acute myeloid leukaemia in first remission: results of MRC AML 10 Trial. Lancet 1998; 351: 700-708. https://doi. org/10.1016/S0140-6736(97)09214-3

[9] CASSILETH PA, HARRINGTON DP, APPELBAUM FR, LAZARUS HM, ROWE JM et al. Chemotherapy compared with autologous or allogeneic bone marrow transplantation in the management of acute myeloid leukemia in first remission. N Engl J Med.1998; 339: 1649-1656. https://doi.org/10.1056/ NEJM199812033392301

[10] VELlENGA E, VAN PUTtEN W, OsSENKOPPELE GJ, VERDONCK LF, THEOBLAD M et al. Autologous peripheral blood stem cell transplantation for acute myeloid leukemia. Blood 2011; 118: 6037-6042. https://doi. org/10.1182/blood-2011-07-370247

[11] ZITTOUN RA, MANDELLI F, WILLEMZE R, DE WITTE T, LABAR B et al. Autologous or allogeneic bone marrow transplantation compared with intensive chemotherapy in acute myelogenous leukemia. European Organization for Research and Treatment of Cancer (EORTC) and the Gruppo Italiano Malattie Ematologiche Malignedell' Adulto (GIMEMA) Leukemia Cooperative Groups. N Engl J Med. 1995; 332: 217-223. https://doi.org/10.1056/NEJM199501263320403

[12] LINKER CA, RIES CA, DAMON LE, SAYRE P, NAVARRO et al. Autologous stems cell transplantation for acute myeloid leukemia in first remission. Biol Blood Marrow Transplant 2000; 6: 50-57. https://doi.org/10.1016/S1083-8791(00)70052-8

[13] MIYAMOTO T, NAGAFUJI K, HARADA M, ETO T, FUJISAKI et al. Quantitative analysis of AML1/ETO transcripts in peripheral blood stem cell harvests from patients with $\mathrm{t}(8 ; 21)$ acute myelogenous leukaemia. Br J Haematol 1995; 91 : 132-138. https://doi.org/10.1111/j.1365-2141.1995.tb05258.x [14] STEIN AS, O'DONNEL MR, CHAI A, SCHMIDT GM, NADEMANEE A et al. In vivo purging with high-dose cytarabine followed by high-dose chemoradiotherapy and reinfusion of unpurged bone marrow for adult acute myelogenous leukemia in first complete remission. J Clin Oncol 1996; 14: 2206-2216. https://doi.org/10.1200/JCO.1996.14.8.2206 
[15] MEHTA J, POWLES R, SINGHAL S, HORTON C, TAIT $\mathrm{D}$ et al. Autologous bone marrow transplantation for acute myeloid leukemia in first remission: identification of modifiable prognostic factors. Bone Marrow Transplant 1995; 16: 499-506.

[16] KORBLING M, FLIEDNER TM, HOLLE R, MAGRIN S, BAUMANN M et al. Autologous blood stem cell (ABSCT) versus purged bone marrow transplantation ( $\mathrm{pABMT}$ ) in standard risk AML: influence of source and cell composition of the autograft on hematopoietic reconstitution and disease-free survival. Bone Marrow Transplant 1991; 7: 343-349.

[17] SANZ MA, RUBIA J, SANZ GF, MARTIN G, MARTINEZ J et al. Busulfan plus cyclophosphamide followed by autologous stem cell transplantation for patients with acute myeloblastic leukemia in first complete remission: a report from a single institution. J Clin Oncol 1993; 11: 1661-1667. https://doi. org/10.1200/JCO.1993.11.9.1661
[18] REIFFERS J, KORBLING M, LABOPIN M, GORIN NC. Autologous blood stem cell transplantation versus autologous bone marrow transplantation for acute myeloid leukemia in first completeremission. The EBMT Group Working Party for Autologous Bone Marrow Transplantation. Bone Marrow Transplant 1991; 7 Suppl 2:144.

[19] LAPORTE JP, GORIN NC, FEUCHTENBAUM J, LOPEZ M, DOUAY L et al. Relapse after autografting with peripheral blood stem cells. Lancet 1987; 2: 1393. https://doi.org/10.1016/ $\underline{\text { S0140-6736(87)91278-5 }}$

[20] GORIN NC, LABOPIN M, CZERW T, PABST T, BLAISE $\mathrm{D}$ et al. Autologous stem cell transplantation for adult acute myelocytic leukemia in first remission-Better outcomes after busulfan and melphalan compared with busulfan and cyclophosphamide: A retrospective study from the Acute Leukemia Working Party of the European Society for Blood and Marrow Transplantation (EBMT). Cancer 2017; 123: 824-831. https:// doi.org/10.1002/cncr.30400 